\title{
Restricting Marketing of Unhealthy Foods: Should General Internists Engage?
}

Norm Campbell MD, Tara Duhaney MHSc

\section{About the Authors}

Norm Campbell (right) is a professor of medicine, community health sciences and physiology and pharmacology at the University of Calgary and is a member of the Libin Cardiovascular Institute, in Calgary, Alberta. Tara Duhaney is a policy director at the Libin Cardiovascular Institute. Correspondence may be directed to ncampbel@ucalgary.ca.

$\mathrm{U}$ nhealthy diet is the leading risk for death, years of life lost, and disability, causing an estimated 65,722 deaths and 864,032 life years lost in Canada in 2010. ${ }^{1}$ Although the causes of unhealthy diet are complex, unhealthy eating habits start early in life, and unhealthy food and beverage marketing to children is consistently associated with unhealthy dietary behaviours and childhood obesity. Although there have been recommendations from the World Health Organization (WHO) and the United Nations urging countries to restrict such marketing to children as a population strategy to improve diet, the food industry continues to direct millions of marketing dollars to increase the sales and consumption of the very foods that contribute to disease burden. While many countries have heeded the WHO recommendations, in Canada, outside of Quebec, the food industry largely self-regulates its marketing of unhealthy food to children, with no government monitoring or oversight. The result is that Canadian children are extensively exposed to marketing of unhealthy food products that would not be allowed in several other countries.

Should Canadian internists accept that children's dietary health is being hijacked by an industry that generates profits by selling the very foods that are making our children and future adults unhealthy? Saying enough is enough, the Canadian Society of Internal Medicine and 23 other health and scientific organizations in Canada have called for policies and processes to stop all marketing of unhealthy foods and beverages to children. ${ }^{2}$ Individual internists can advocate through local, provincial, and national organizations, and in our communications with provincial and federal politicians. The role of unhealthy foods as the major cause of death and disability needs to be part of undergraduate and continuing education, and other educational opportunities focused on health promotion and disease prevention.

\section{References}

1. Institute for Health Metrics and Evaluation. Global burden of disease arrow diagram. Seattle (WA): The Institute, 2013;

http://www.healthmetricsandevaluation.org/gbd/visualizations/gbd-arrowdiagram. Accessed September 10, 2013.

2. Canadian Hypertension Advisory Committee. Restricting marketing of unhealthy foods and beverages to children and youth in Canada. Calgary (AB): The Committee, 2013; http://www.hypertensiontalk.com/publications/marketing_to_children/. Accessed September 10, 2013. 